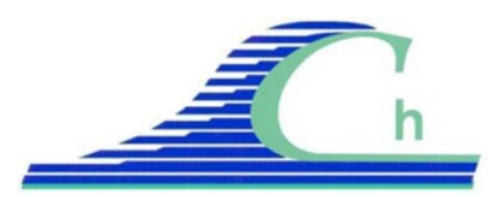

XII èmes Journées Nationales Génie Côtier - Génie Civil

Cherbourg, 12-14 juin 2012

DOI:10.5150/jngcgc.2012.103-L C Editions Paralia CFL

disponible en ligne - http://www.paralia.fr - available online

\title{
Évolution de l'emprise des zones inondables de l'estuaire de la Gironde sous l'influence du changement climatique
}

\author{
Vanessya LABORIE ${ }^{1,2}$, François HISSEL ${ }^{3}$, Philippe SERGENT ${ }^{4}$
}

1. CETMEF - 2, boulevard GAMBETTA BP 60039, 60321 Compiègne Cedex, France.

2. Laboratoire Saint-Venant (EDF R\&D, CETMEF, École des Ponts ParisTech), 6, quai Watier, BP49, 78401 Chatou, France.

vanessya.laborie@developpement-durable.gouv.fr

3. CETMEF - 2, boulevard GAMBETTA BP 60039, 60321 Compiègne Cedex, France. francois.hissel@developpement-durable.gouv.fr

4. CETMEF - 2, boulevard GAMBETTA BP 60039, 60321 Compiègne Cedex, France. philippe.sergent@developpement-durable.gouv.fr

\section{Résumé :}

Dans le cadre du projet européen THESEUS, un modèle avec débordement de l'estuaire de la Gironde a été utilisé pour cartographier l'évolution de l'emprise des zones inondables et des quantiles de niveaux d'eau à l'horizon 2100 sur l'ensemble de l'estuaire en utilisant l'hypothèse pessimiste d'élévation du niveau de la mer de l'ONERC (60 cm à l'horizon 2100). Les données d'entrée du modèle sont les champs de vent à Royan et Mérignac, le signal global au Verdon et les débits des 3 rivières (Garonne, Dordogne et Isle). Un modèle simplifié de calcul des surcotes à partir des champs de vent et de pression du CLM/SGA a été construit et validé au Verdon. Ainsi alimenté, le modèle a permis de construire les cartes d'inondation correspondant aux périodes de retour de 2 à 100 ans pour les périodes [1960 ; 1999], [2010 ; 2039], [2040;2069] et [2070; 2099] sur 3 sites particuliers de l'estuaire.

Compte-tenu des hypothèses faites, l'impact du changement climatique sur l'évolution de l'emprise des zones inondées dans l'estuaire de la Gironde et sur les quantiles de hauteur d'eau dans le lit majeur dépend principalement de l'élévation du niveau de la mer sur la période considérée. Des zones qui ne sont aujourd'hui pas inondées pour de faibles périodes de retour le deviennent à l'horizon 2100. La zone du Verdon ne subit un impact négatif qu'à moyen et long terme. Au droit de Bordeaux, des effets sensibles se feraient sentir le long de la route nationale RN230 à l'horizon 2100. L'influence des débits et des ruptures de digues devra être étudiée afin de préciser ces résultats.

\section{Mots-clés :}

Estuaire de la Gironde - Changement climatique - Niveaux d'eau extrêmes - Surcotes Cartographie - Zones inondables - Méthode statistique du renouvellement

\section{Introduction}

Dans le cadre du projet Européen THESEUS ("Innovative technologies for safer 
European coasts in a changing climate"), dont l'objectif est de mettre au point des structures de protection côtière innovantes dans un contexte de changement climatique, les niveaux d'eau extrêmes et l'évolution de l'emprise des champs d'expansion de crue à l'horizon 2100 ont été étudiés sur l'estuaire de la Gironde.

\section{Localisation du site d'étude et description du modèle}

Pour produire des cartes des niveaux d'eau sur chaque point de l'estuaire de la Gironde pour des périodes de retour de 2 à 100 ans à l'horizon 2100 et évaluer l'évolution des quantiles de niveaux d'eau dans le lit majeur de l'estuaire à court, moyen et long termes par rapport à la période actuelle, un modèle numérique bidimensionnel et fondé sur les équations de Saint-Venant de l'estuaire de la Gironde a été utilisé (LABORIE et al., 2012).

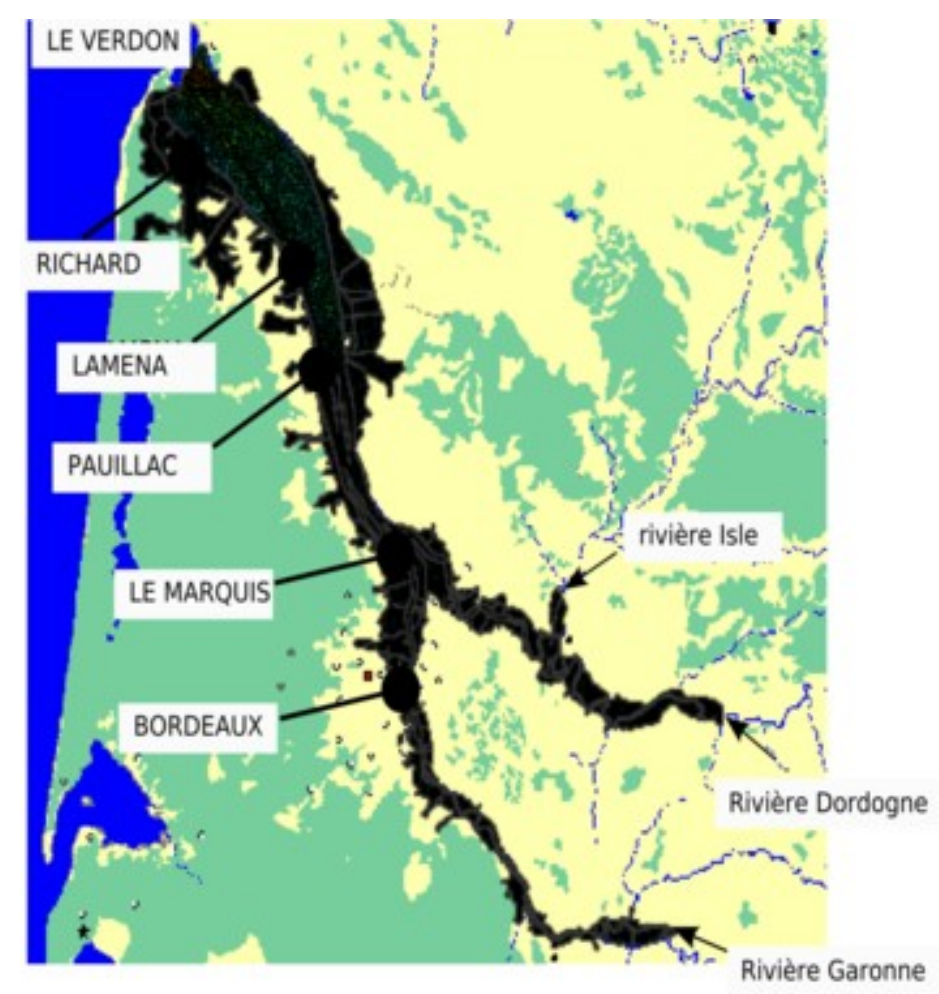

Figure 1. Localisation et emprise du modèle de l'estuaire de la Gironde.

Le modèle couvre l'ensemble de l'estuaire depuis la Réole sur la Garonne et Pessac sur la Dordogne. Sa frontière maritime se situe à la Pointe du Verdon. A l'amont, il prend en compte les débits d'apport de l'Isle à Libourne au droit de sa confluence avec la Dordogne. Il présente la particularité de prendre en compte les débordements du lit mineur de l'estuaire ou des quatre rivières (Dordogne, Garonne, Isle, Dronne) vers le lit majeur. Son emprise est représentée sur la figure 1 ci-dessus. Il s'étend sur $115 \mathrm{~km}$ environ d'est en ouest. 


\section{XII ${ }^{\text {èmes }}$ Journées Nationales Génie Côtier - Génie Civil \\ Cherbourg, 12-14 juin 2012}

\section{Données et paramètres de simulation}

Des données d'entrée ont été rassemblées afin d'alimenter le modèle entre 1960 et 2100.

\subsection{Champs de vent et de pression et scénario climatique étudié}

Les vents utilisés dans le cadre de cette étude sont fournis par le modèle climatologique européen CLM/SGA, développé par le Max Planck Institut (MPI). Une période de référence et un scénario de changement climatique ont été utilisés : C20 pour la période [1960 ; 2000] et A1B pour la période [2010 ; 2100].

\section{2 Élévation du niveau de la mer}

Le scénario de changement climatique décrit dans le paragraphe précédent a été associé à l'hypothèse pessimiste d'élévation du niveau de la mer (60 cm en 2100) de l' Observatoire National sur les Effets du Réchauffement Climatique (ONERC, 2010).

\subsection{Débits fluviaux sur l'Isle, la Garonne et la Dordogne}

Les débits de la Garonne et de la Dordogne sur la partie amont du modèle ont été fournis par le Grand Port de Bordeaux pour la période [1960 ; 2009]. Ils ont été recalés de telle sorte que, pour la période passée [1960-2000], les quantiles de niveaux d'eau au Marquis et à Bordeaux (tous deux sous influence fluviale) soient cohérents avec la bibliographie sur le sujet (en particulier, (SOGREAH, 2010)). Pour la période [2010 ; 2100], les chroniques passées des débits ainsi recalés ont été rejouées, sans prendre en compte les effets du changement climatique. En ce qui concerne l'Isle, ce sont les débits mensuels issus de la banque HYDRO qui ont alimenté le modèle.

\subsection{Construction et validation d'un modèle empirique de surcotes au Verdon}

Le signal global à la frontière maritime du Verdon est la somme de deux composantes : la marée prédite obtenue grâce au code de calcul PREDITC (développé par le SHOM) et la surcote météorologique, obtenue à l'aide du modèle empirique de surcotes construit et validé dans le cadre de cette étude et décrit ci-dessous.

Ainsi, en utilisant les champs de vent et de pression du modèle climatologique CLM/SGA moyennés sur l'emprise du modèle et la relation de régression suivante :

$\delta=a_{C L M} * v_{m o y}{ }^{2}+b_{C L M} * p_{m o y}+c_{C L M}$

où :

$\delta$ = surcote instantanée maximale sur 3 h (m);

$v_{\text {moy }}=$ norme moyenne de la vitesse du vent sur l'ensemble de l'estuaire de la Gironde ;

$p_{\text {moy }}=$ pression moyenne sur l'estuaire de la Gironde :

$a_{C L M}, b_{C L M}$ and $c_{C L M}=$ coefficients obtenus par ajustement sur les quantiles statistiques de niveaux d'eau au Verdon $\left(a_{C L M}=3,32 \times 10^{-3} ; b_{C L M}=-9,95 \times 10^{-5} ; c_{C L M}=9,89\right)$, le signal global (marée + surcote) injecté dans le modèle numérique au Verdon sur la 
période [1960 ; 2000] vérifie la même distribution statistique que celle obtenue en utilisant les mesures marégraphiques et décrite dans (SOGREAH, 2010).

Sur la période [1960 ; 2100] le signal de surcotes au Verdon a été produit en utilisant la relation (1) qui relie les champs de vent et de pression du modèle climatologique européen CLM/SGA à la surcote météorologique au Verdon. Le signal global composé de la marée prédite et de la surcote est alors imposé sur la frontière maritime du modèle numérique de l'estuaire de la Gironde.

Les normes des champs de vitesses fournis par le modèle CLM/SGA du MPI décroissent ou subissent une évolution peu prononcée d'ici 2100 sur la plus grande partie de la façade méditerranéenne et sur le golfe de Gascogne (IMGW et al., 2011). Les surcotes météorologiques issues du modèle simplifié sont corrélées aux vitesses du vent, il n'est ainsi pas étonnant que les quantiles de surcotes au Verdon suivent cette même tendance (décroissante) à l'horizon 2100 (LABORIE et al., 2012). En conséquence, l'élévation du niveau de la mer est directement responsable de l'augmentation des quantiles de niveaux d'eau à l'intérieur de l'estuaire de la Gironde et, en particulier, à sa frontière maritime, située au Verdon.

\section{Cartographie des cartes d'inondation à l'horizon 2100}

\subsection{Calcul des quantiles en chaque point de l'estuaire de la Gironde}

Une méthode du renouvellement a été utilisée en chaque nœud du maillage du modèle numérique de l'estuaire de la Gironde pour obtenir les quantiles correspondant à des périodes de retour de 1 à 100 ans.

Pour des périodes de retour inférieures à 30 ans (2, 5, 10 et 20 ans), les quantiles statistiques ont été utilisés directement. Pour des périodes de retour supérieures à 50 ans (50 et 100 ans), un ajustement à une loi de Gumbel a été utilisé.

4.2 Impact du changement climatique sur les zones inondables de l'estuaire de la Gironde

Du fait de l'étendue du secteur d'étude, les hauteurs d'eau correspondant aux périodes de retour de 2 à 100 ans ont été cartographiées sur 3 sites particuliers de l'estuaire situés : à la frontière maritime de l'estuaire autour du Verdon ; à la confluence entre la Dordogne et la Garonne, au niveau de la presqu'île d'Ambès ; autour de Bordeaux.

\section{a) Analyse des résultats à la frontière maritime du modèle dans la région du Verdon}

Autour du Verdon, les quantiles de hauteur d'eau pour une période de retour de 100 ans dans les conditions actuelles (en haut à gauche) et à court ([2010;2039] en haut à droite), moyen ([2040 ; 2069] en bas à gauche) et long ([2070 ; 2099] en bas à droite) termes sont représentés sur la figure 2 ci-dessous. 


\section{XII èmes Journées Nationales Génie Côtier - Génie Civil \\ Cherbourg, 12-14 juin 2012}
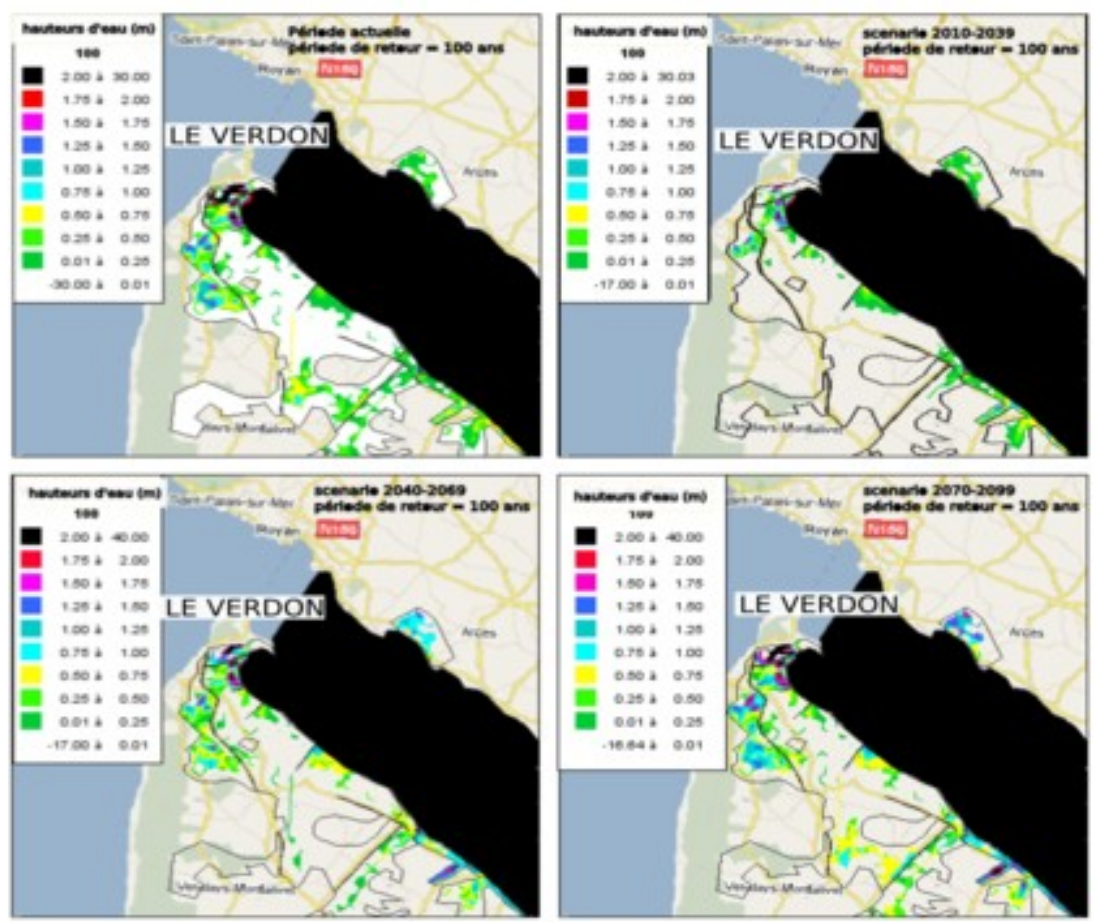

Figure 2. Quantiles de hauteur d'eau simulés pour une période de retour de 100 ans dans la région du Verdon pour la période actuelle et à court, moyen et long terme.

Sur la période [2010 ; 2039], en rive gauche de l'embouchure de l'estuaire, malgré une élévation du niveau de la mer en moyenne égale à $9 \mathrm{~cm}$, la diminution des quantiles de surcotes conduit à une diminution des quantiles de niveau d'eau dans le champ d'expansion des crues et de l'emprise de celui-ci. Des zones inondées pour une période de retour de 2 ans actuellement, en particulier au droit des axes routiers, zones d'accumulation naturelle des écoulements, ne le sont plus.

Les tendances sont assez similaires sur la période [2040 ; 2069]. En effet, l'emprise du lit majeur diminue malgré l'élévation du niveau moyen de la mer (39 cm en 2070). Certaines zones inondées par 50 à $75 \mathrm{~cm}$ d'eau pour une période de retour de 100 ans ne sont plus inondées.

Sur la période [2070 ; 2099], pour laquelle l'élévation du niveau de la mer est la plus forte (60 cm à l'horizon 2100), l'emprise du champ d'expansion de crues est identique à celle de la période actuelle, mais avec des hauteurs d'eau bien plus importantes (jusqu'à 25 à 50 cm d'augmentation pour une période de retour de 100 ans).

\section{b) Analyse des résultats à la confluence entre la Dordogne et la Garonne}

Les quantiles de hauteur d'eau pour une période de retour de 100 ans obtenus pour ce secteur d'étude sont représentés sur la figure 3. 

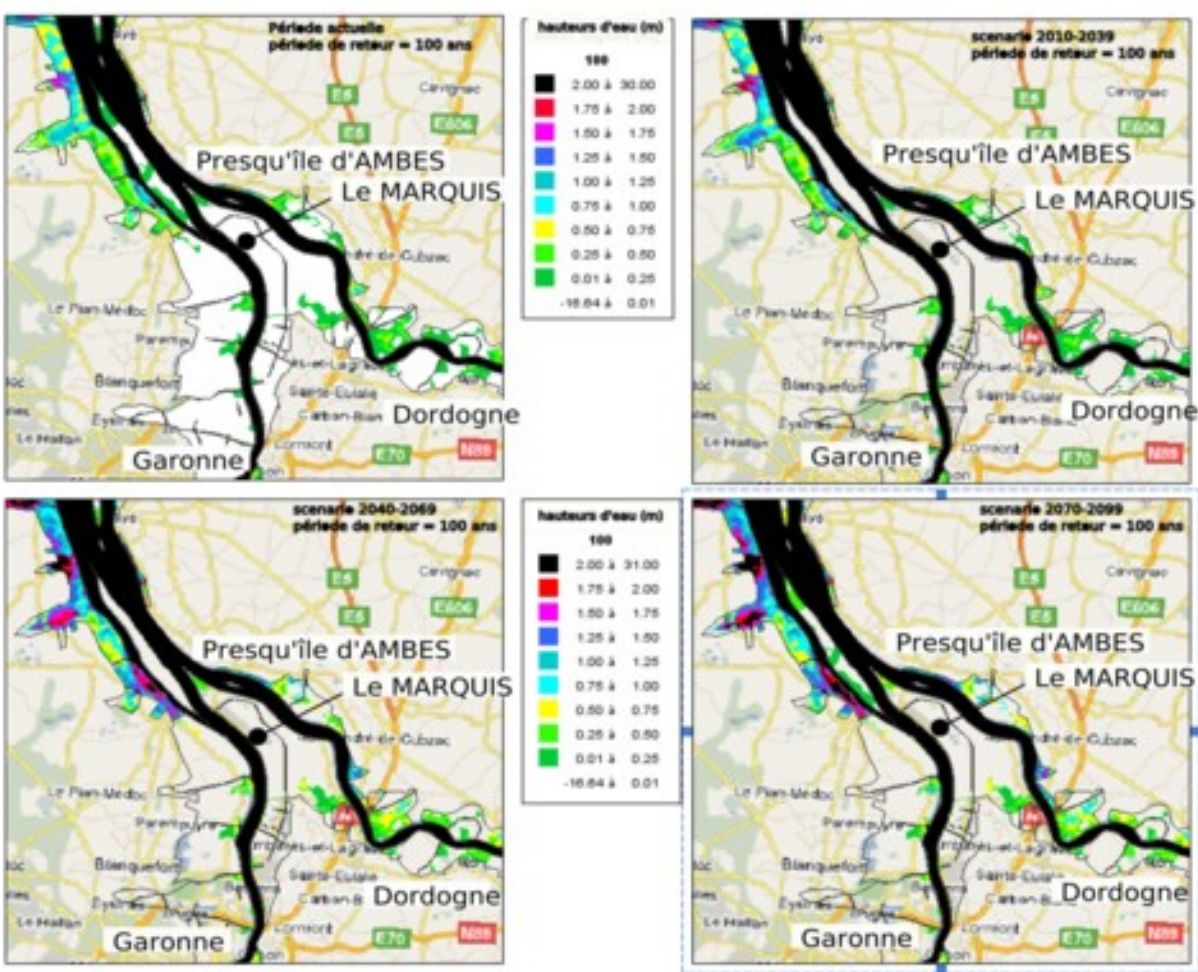

Figure 3. Quantiles de hauteur d'eau simulés dans la région d'Ambès (confluence entre la Dordogne et la Garonne) pour la période actuelle et à court, moyen et long termes.

A court et moyen termes, aucun changement significatif n'apparaît à l'amont de la confluence. Ce secteur d'étude subit l'influence des régimes hydrologiques de la Dordogne et de la Garonne dont les débits ont été ajustés et fortement réduits de telle sorte que les quantiles à Bordeaux et au Marquis soient cohérents avec la littérature sur le sujet. L'influence des débits devra donc être étudiée pour préciser ces résultats.

Les hauteurs d'eau dans le champ d'expansion des crues de la Dordogne restent, en termes de quantiles, identiques à la période de référence à court terme. C'est seulement à moyen et long termes qu'elles évoluent, notamment en rive droite de la Dordogne où les quantiles augmentent d'environ $25 \mathrm{~cm}$.

En aval de la confluence, les quantiles de hauteur d'eau subissent une augmentation pour toutes les périodes de retour : de 25 à $50 \mathrm{~cm}$ pour la période [2010;2039], de 50 cm à 75 cm sur [2040;2069] et de plus de 75 cm à long terme.

A court et moyen termes, les chemins empruntés par les écoulements traversant la presqu'île d'Ambès disparaissent et ne réapparaissent qu'à long terme, sans modification des hauteurs d'eau.

c) Évolution des zones inondables dans la région de Bordeaux.

Dans la région de Bordeaux, les quantiles de hauteurs d'eau correspondant à une période de retour de 100 ans sont représentés sur la figure 4 . 


\section{XII ${ }^{\text {èmes }}$ Journées Nationales Génie Côtier - Génie Civil \\ Cherbourg, 12-14 juin 2012}

Dans cette zone, les résultats sont fortement influencés par la diminution du débit d'apport de la Garonne (au niveau de la Réole) tel que choisi dans le modèle et il est possible que les quantiles de hauteur d'eau sur la rive droite du méandre de la Garonne, au droit de Bordeaux, soient sous-estimés par notre méthode et nos hypothèses.

A l'horizon 2100, le lit majeur de la Garonne n'évolue pas significativement.

Au droit de Bordeaux, les quantiles de hauteur d'eau augmentent cependant de $80 \mathrm{~cm}$ le long de la route nationale RN230 pour une période de retour de 100 ans. Pour une période de retour de 2 ans, ce secteur devient inondable à court terme. Les quantiles de hauteurs d'eau augmentent d'environ $50 \mathrm{~cm}$ à l'horizon 2100.

Les zones encaissées se remplissent un peu plus du fait de l'impact de l'élévation du niveau de la mer dans le lit mineur (LABORIE et al., 2012) et concentrent ainsi les masses d'eau supplémentaires lors des événements débordants.

Sur la rive gauche de la Garonne, la région située au sud immédiat de Villenave d'Ornon subit également un fort impact du changement climatique. En effet, le champ d'expansion des crues s'étend pour une période de retour de 2 ans et les quantiles de hauteur d'eau augmentent d'environ $25 \mathrm{~cm}$ à l'horizon 2100. L'augmentation des quantiles est plus prononcée à moyen terme et il y a lieu de s'interroger sur le rôle que jouent les chroniques de débits utilisées dans ces résultats. La rive droite de la Garonne est également particulièrement impactée dans la périphérie de l’’le de la Lande.
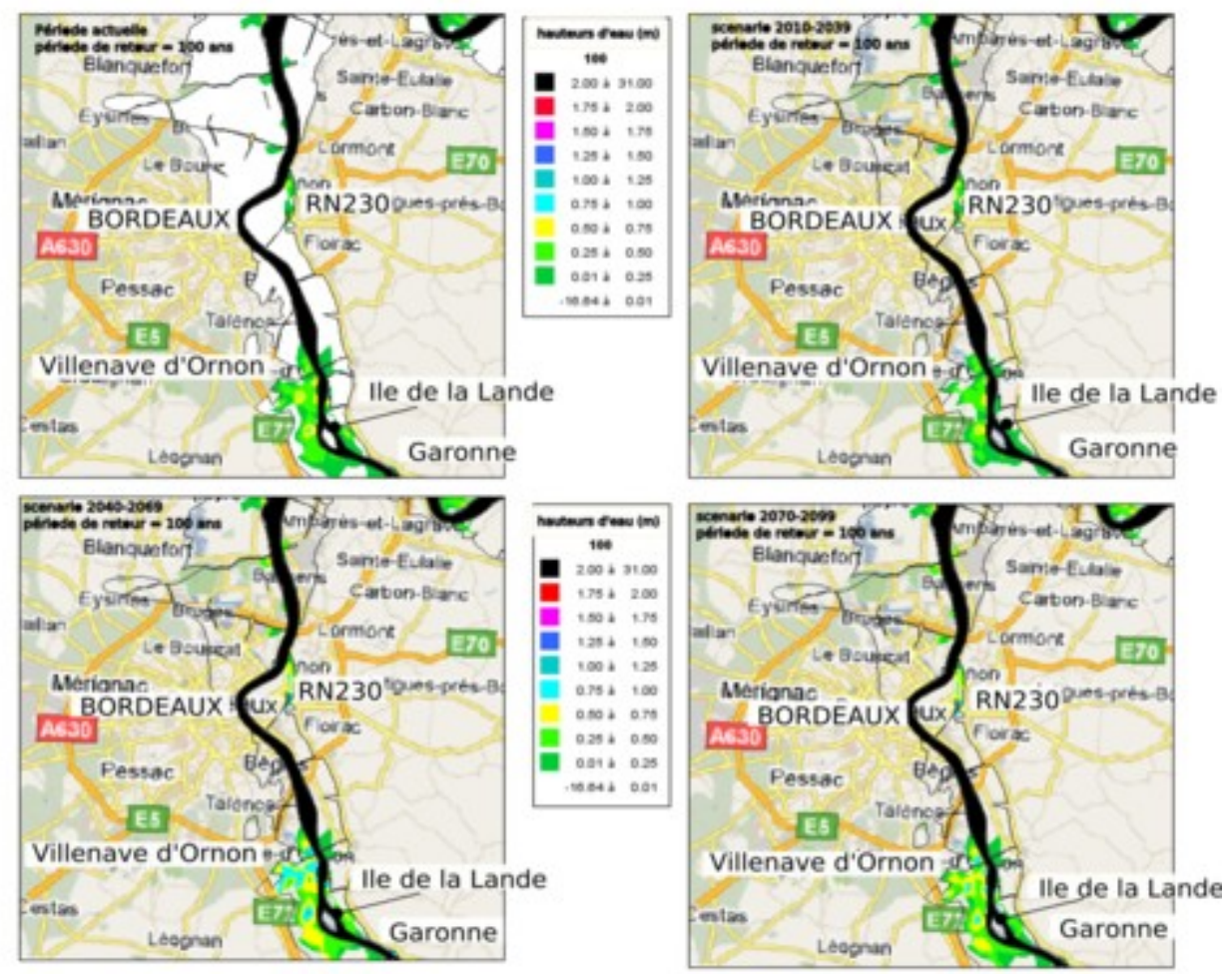

Figure 4. Quantiles de hauteur d'eau simulés pour une période de retour de 100 ans dans la région de Bordeaux pour la période actuelle et à court, moyen et long termes. 


\section{Conclusions et perspectives}

Un modèle numérique a été utilisé pour simuler les niveaux d'eau atteints dans le champ d'expansion des crues de l'estuaire de la Gironde pour la période actuelle et à court, moyen et long termes. A l'horizon 2100, l'impact du scénario de changement climatique étudié est important et des régions actuellement non situées en zone inondable pour une période de retour de 2 ans le deviennent.

Il apparaît que la région du Verdon subit un impact à moyen et long termes. Pour la période [2010; 2039], une légère diminution de l'emprise du lit majeur et des quantiles peut être observée. Des changements significatifs sont observés au droit de Bordeaux, en rive droite le long de la RN230, et sur la rive gauche au sud de Villenave d'Ornon.

Pour affiner cette analyse, les effets liés aux chroniques de débits utilisées et aux ruptures de digues doivent être étudiés.

\section{Remerciements}

Les auteurs tiennent à adresser leurs remerciements au Centre d'Etudes Techniques de l'Equipement du Sud-Ouest, aux Services de Prévision des Crues Littoral Atlantique et Dordogne, à SOGREAH et à METEO-FRANCE.

Cette étude a lieu dans le cadre du projet européen THESEUS.

\section{Références bibliographiques}

IMGW, IBW PAN, MHI, UC, IORAS, CETMEF, UoP, CORILA, IO-BAS, BRGM, K.U.LEUVEN (2011), Database including recent characteristics of regional and local climate variations and extreme events associated with climate change, THESEUS Internal Deliverable 1.11, $31 \mathrm{p}$.

LABORIE V., HISSEL F., SERGENT P. (2012). Évaluation des niveaux d'eau extrêmes du futur sur l'estuaire de la Gironde. Colloque SHF "événements extrêmes fluviaux et maritimes", Paris, 8 p.

ONERC -Observatoire National sur les Effets du Réchauffement Climatique- (2010). Prise en compte de l'élévation du niveau de la mer en vue de l'estimation des impacts $d u$ changement climatique et des mesures d'adaptation possibles. Synthèse $n^{\circ}$ 2, 6 p.

SOGREAH (2010). Référentiel de protection contre les inondations le long de l'estuaire de la Gironde, de la Garonne et de la Dordogne, Rapport d'étape 4 : définition des événements de référence, Version I. Rapport n 4310990-1 74 0638, 199 p. 